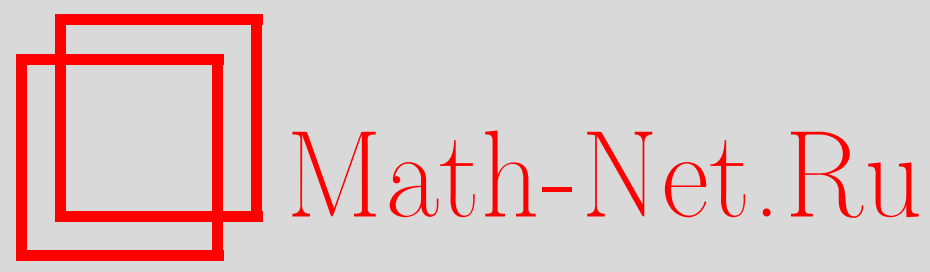

В. В. Костин, Замкнутость справа мартингальных последовательностей в смысле $A$-интеграла, Матем. заметки, 2000, том 68, выпуск 1, 98-104

DOI: https://doi.org/10.4213/mzm923

Использование Общероссийского математического портала Math-Net.Ru подразумевает, что вы прочитали и согласны с пользовательским соглашением http://www.mathnet.ru/rus/agreement

Параметры загрузки:

IP : 54.209 .52 .79

26 апреля 2023 г., 13:21:41 


\title{
ЗАМКНУТОСТЬ СПРАВА МАРТИНГАЛЬНЫХ ПОСЛЕДОВАТЕЛЬНОСТЕЙ В СМЫСЛЕ $A$-ИНТЕГРАЛА
}

\section{В.В. Костин}

\begin{abstract}
В этой работе даются новые достаточные (а для мартингалов особого вида необходимые и достаточные) условия замкнутости мартингалов справав смысле $A$-интеграла. Эти результаты следуют из доказанной в работе теоремы о предельном переходе под знаком $A$-интеграла. Теорема установлена с помощью критерия перестановки двух пределов по базе. Показьвается, что полученные таким образом достаточные условия сильнее известных ранее.
\end{abstract}

Библиография: 4 названий.

Предельные теоремы для мартингалов являются важным инструментом в теории рядов по некоторым классическим системам (Хаара, Уолша, мультипликативньп системам Виленкина и др.). А именно, каждая теорема, дающая достаточные условия замкнутости справа для мартингалов, автоматически дает достаточные условия принадлежности рядов по таким системам к классу рядов Фурье, и при этом последние элементы мартингалов и служат образующими эти ряды функциями (см. теорему 1 из [1]). Эта теорема остается верной и тогда, когда используемые в ней термины понимаются в обобщенном смысле, т. е. когда в их определении вместо интеграла Лебега участвует какой-либо другой интеграл.

Здесь мы рассматриваем замкнутость мартингалов справа в смысле $A$-интеграла, который впервые был введен А. Н. Колмогоровым в [2].

Напомним некоторые определения.

ОПРЕДЕЛЕНИЕ 1. Пусть $(\Omega, \mathscr{B}, \mathrm{P})$ - вероятностное пространство, на нем определена случайная величина $X$. Тогда $X$ называется $A$-интегрируемой на множестве $B \in \mathscr{B}$, если вьполняются следующие условия:

1. $\mathrm{P}\{B \cap\{|X|>C\}\}=\overline{\bar{o}}(1 / C), C \rightarrow \infty$;

2. существует $\lim _{C \rightarrow \infty} \int_{B}[X]^{C} d P$.

Этот предел назьвается $A$-интегралом случайной величины $X$ по множеству $B$ и обозначается $(A) \int_{B} X d P$. Здесь через $[X]^{C}$ обозначена срезка функции $X$, определяемая так $(C \geqslant 0)$ :

$$
[X]^{C}= \begin{cases}C, & X>C, \\ X, & -C \leqslant X \leqslant C, \\ -C, & X<-C .\end{cases}
$$

Работа выполнена при поддержке Российского фонда фундаментальных исследований, гранты № 96-01-00332 и № 96/97-15-96073. 
Определим также срезки другого типа:

$$
[X]_{C}= \begin{cases}X, & -C \leqslant X \leqslant C, \\ 0 & \text { иначе. }\end{cases}
$$

Понятно, что в силу 1 -го условия значение $A$-интеграла не зависит от типа применяемой срезки.

ОПрЕДЕЛЕниЕ 2 . Пусть $X-A$-интегрируемая случайная величина, определенная на $(\Omega, \mathscr{B}, \mathrm{P}), \sigma$-алгебра $\mathscr{F}$ - подалгебра алгебры $\mathscr{B}$. Обобщенным условным математическим ожиданием (в смысле $A$-интеграла) случайной величины $X$ относительно $\sigma$-алгебры $\mathscr{F}$ называется случайная величина $A E(X, \mathscr{F})$ такая, что

1. $A E(X, \mathscr{F})$ измерима относительно $\mathscr{F}$;

2. $\forall B \in \mathscr{F}$ вьполнено $(A) \int_{B} X d P=\int_{B} A E(X, \mathscr{F}) d P$.

$A$-интеграл шире интеграла Лебега, но если $\mathscr{G}$-измеримая случайная величина $Y$ $A$-интегрируема на любом множестве $B \in \mathscr{G}$, то она $A$-интегрируема и на множестваx $\{Y>0\},\{Y \leqslant 0\}$, а тогда она, как нетрудно видеть, $L$-интегрируема, и поэтому в определении $A E(X, \mathscr{F})$ в условии 2 справа достаточно писать интеграл Лебега.

ОПРЕДЕЛЕНИЕ 3 . Пусть задано вероятностное пространство $(\Omega, \mathscr{B}, \mathrm{P})$ с фильтрацией, т. е. семейством $\left\{\mathscr{F}_{n}\right\} \quad \sigma$-алгебр $\mathscr{F}_{n}, n \geqslant 1$, таких, что $\mathscr{F}_{1} \subseteq \cdots \subseteq \mathscr{F}_{n} \subseteq \mathscr{F}_{n+1}$ $\subseteq \cdots \subseteq \mathscr{B}$. Последовательность $\left\{X_{n}(\omega), \mathscr{F}_{n}\right\}_{n=1}^{\infty}$ случайных величин $X_{n}$, измеримых относительно соответствующих $\sigma$-алгебр $\mathscr{F}_{n}$, называется мартингальной (в смысле $A$-интеграла), если $\forall m, n, m \geqslant n$, вьполнено $X_{n}=A E\left(X_{m}, \mathscr{F}_{n}\right)$, при этом она называется замкнутой справа последним әлементом $X_{\infty}$, если $\forall n$ выполнено $X_{n}=$ $A E\left(X_{\infty}, \mathscr{F}_{n}\right)$.

В силу вьшеприведенного замечания определения последовательностей, мартингальных в смысле $A$-интеграла и мартингальных в обычном смысле, т. е. когда при определении условного математического ожидания вместо $A$-интеграла берется интеграл Лебега, совпадают. Различие интегралов проявляется лишь в определении замкнутости справа.

Основным результатом статьи является следующая теорема, дающая при некоторых естественных предположениях необходимые и достаточные условия замкнутости справа мартингальной последовательности.

ТЕОремА 1. Пусть $\left\{X_{n}(\omega), \mathscr{F}_{n}\right\}_{n=1}^{\infty}-$ мартингальная последовательность, определенная на вероятностном пространстве $(\Omega, \mathscr{B}, \mathrm{P})$, такая, что $X_{n}$ сходится по мере к случайной величине $X_{\infty}$ и удовлетворяет следующему условию:

$$
\forall \varepsilon>0 \quad \exists C_{\varepsilon} \quad \forall C \geqslant C_{\varepsilon} \quad \exists N_{C} \quad \forall n \geqslant N_{C} \quad C \mathrm{P}\left\{\left|X_{n}\right|>C\right\}<\varepsilon .
$$

При этих условиях $X_{\infty}$ на каждом $B \in \bigcup_{n} \mathscr{F}_{n}$ A-интегрируема и является последним әлементом мартингальной последовательности $\left\{X_{n}, \mathscr{F}_{n}\right\}$ тогда и только тогда, когда $\forall B \in \bigcup_{n} \mathscr{F}_{n}$ выполнено

$$
\forall \varepsilon>0 \quad \exists C_{\varepsilon} \quad \forall C \geqslant C_{\varepsilon} \quad \exists N_{C} \quad \forall n \geqslant N_{C} \quad\left|\int_{B \cap\left\{\left|X_{n}\right|>C\right\}} X_{n} d P\right|<\varepsilon .
$$

Эта теорема будет получена из следующей теоремы для предельного перехода под знаком $A$-интеграла. 
ТеОрема 2. Пусть $\left\{X_{n}(\omega)\right\}_{n=1}^{\infty}-$ последовательность $A$-интегрируемых случайных величин, определенных на $(\Omega, \mathscr{B}, \mathrm{P})$, сходящаяся по мере к случайной величине $X_{\infty}$ и такая, что

$$
\forall \varepsilon>0 \quad \exists C_{\varepsilon} \quad \forall C \geqslant C_{\varepsilon} \quad \exists N_{C} \quad \forall n \geqslant N_{C} \quad C \mathrm{P}\left\{\left|X_{n}\right|>C\right\}<\varepsilon .
$$

Тогда следующие условия эквивалентны:

1. $X_{\infty}$ является $A$-интегрируемой и существует предел

$$
\lim _{n \rightarrow \infty}(A) \int X_{n} d P=(A) \int X_{\infty} d P
$$

2.

$$
\forall \varepsilon>0 \quad \exists C_{\varepsilon} \quad \forall C \geqslant C_{\varepsilon} \quad \exists N_{C} \quad \forall n \geqslant N_{C} \quad\left|(A) \int_{\left\{\left|X_{n}\right|>C\right\}} X_{n} d P\right|<\varepsilon .
$$

Предварительно докажем следующее простое утверждение.

Лемма. Если $X_{n} \rightarrow X_{\infty}$ nо мере, то $u\left[X_{n}\right]^{C} \rightarrow\left[X_{\infty}\right]^{C}$ nо мере $\forall C$.

ДокАЗАтЕЛьСтво. Для $C=0$ утверждение очевидно. Пусть $C>0$. Возьмем произвольное $\varepsilon>0$. Разобьем $\Omega$ на 3 части в зависимости от значений $X_{\infty}: \Omega=\bigcup_{i=1}^{3} \Omega_{i}:=$ $\left\{X_{\infty}<-C\right\} \cup\left\{-C \leqslant X_{\infty} \leqslant C\right\} \cup\left\{X_{\infty}>C\right\}$. Возьмем произвольное $\varepsilon>0$. Выберем $C^{\prime}>C$ такое, что $\mathrm{P}\left\{C<X_{\infty} \leqslant C^{\prime}\right\}<\varepsilon / 5$. Пусть $\delta:=\min \left\{C-C^{\prime}, \varepsilon\right\}$. В силу сходимости $X_{n} \rightarrow X_{\infty}$ по мере

$$
\exists N \quad \forall n \geqslant N \quad \mathrm{P}\left\{\left|X_{n}-X_{\infty}\right|>\delta\right\}<\frac{\varepsilon}{5} .
$$

Тогда для $\Omega_{3}$ (для $\Omega_{1}$ аналогично) имеем

$$
\begin{aligned}
& \mathrm{P}\left\{\left|\left[X_{n}\right]^{C}-C\right|>\varepsilon, X_{\infty}>C\right\}=\mathrm{P}\left\{\left|X_{n}-C\right|>\varepsilon, X_{n}<C, X_{\infty}>C\right\} \\
& \quad \leqslant \mathrm{P}\left\{X_{n}<C, X_{\infty}>C\right\} \leqslant \mathrm{P}\left\{X_{n}<C, X_{\infty}>C^{\prime}\right\}+\mathrm{P}\left\{C<X_{\infty} \leqslant C^{\prime}\right\} \\
& \quad \leqslant \mathrm{P}\left\{X_{\infty}-X_{n}>C^{\prime}-C\right\}+\frac{\varepsilon}{5}<\frac{\varepsilon}{5}+\frac{\varepsilon}{5}=\frac{2 \varepsilon}{5} .
\end{aligned}
$$

Для $\Omega_{2}$ имеем

$$
\mathrm{P}\left\{\left|\left[X_{n}\right]^{C}-\left[X_{\infty}\right]^{C}\right|>\varepsilon,-C \leqslant X_{\infty} \leqslant C\right\} \leqslant \mathrm{P}\left\{\left|X_{n}-X_{\infty}\right|>\varepsilon,-C \leqslant X_{\infty} \leqslant C\right\}<\frac{\varepsilon}{5} .
$$

Суммируя, получаем

$$
\mathrm{P}\left\{\left|\left[X_{n}\right]^{C}-\left[X_{\infty}\right]^{C}\right|>\varepsilon\right\}=\sum_{i=1}^{3} \mathrm{P}\left\{\left|\left[X_{n}\right]^{C}-\left[X_{\infty}\right]^{C}\right|>\varepsilon, \Omega_{i}\right\}<\frac{2 \varepsilon}{5}+\frac{\varepsilon}{5}+\frac{2 \varepsilon}{5}=\varepsilon
$$

что и доказьвает лемму. 
ДоКАЗАТЕЛЬСТво ТЕОРЕмы 2. Докажем сначала, что из условия 1 теоремы следует 1-е условие $A$-интегрируемости $X_{\infty}$. Так как $X_{n} \rightarrow X_{\infty}$ по мере, выберем подпоследовательность $X_{k_{n}} \rightarrow X_{\infty}$ почти всюду. Введем множества $D_{n}(C):=\left\{\left|X_{k_{n}}\right|>C\right\}$, $D(C):=\left\{\left|X_{\infty}\right|>C\right\}, G_{n}(C):=\bigcap_{m=n}^{\infty} D_{m}(C)$. Из сходимости $X_{k_{n}}$ к $X_{\infty}$ п. в. следует, что $D(C)=\bigcup_{n=1}^{\infty} G_{n}(C)$, и так как $G_{n}(C) \subseteq G_{n+1}(C)$, по свойству монотонности вероятности получаем $\mathrm{P}\{D(C)\}=\mathrm{P}\left\{\bigcup_{n=1}^{\infty} G_{n}(C)\right\}=\lim _{n \rightarrow \infty} \mathrm{P}\left\{G_{n}(C)\right\}$. Из условия 1 теоремы $\forall \varepsilon>0 \exists C_{\varepsilon} \forall C \geqslant C_{\varepsilon} \exists N_{C} \forall n \geqslant N_{C} \mathrm{P}\left\{\left|X_{k_{n}}\right|>C\right\}<\varepsilon / C$, а поэтому

$$
\mathrm{P}\{D(C)\}=\lim _{n \rightarrow \infty} \mathrm{P}\left\{G_{n}(C)\right\} \leqslant \lim _{n \rightarrow \infty} \mathrm{P}\left\{D_{n}(C)\right\}<\frac{\varepsilon}{C}
$$

Так как из (1) и (2) следует, что $\forall \varepsilon>0 \exists C_{\varepsilon} \forall C \geqslant C_{\varepsilon} \exists N_{C} \forall n \geqslant N_{C} C \mathrm{P}\left\{\left|X_{n}\right|>C\right\}$ $<\varepsilon / 2$ и $\left|(A) \int X_{n} d P-\int\left[X_{n}\right]_{C} d P\right|<\varepsilon / 2$, то можно оценить

$$
\begin{aligned}
\mid(A) & \int X_{n} d P-\int\left[X_{n}\right]^{C} d P \mid \\
= & \left|(A) \int X_{n} d P-\int\left[X_{n}\right]_{C} d P-\left(C \mathrm{P}\left\{X_{n}>C\right\}-C \mathrm{P}\left\{X_{n}<-C\right\}\right)\right| \\
\leqslant & \left|(A) \int X_{n} d P-\int\left[X_{n}\right]_{C} d P\right|+C \mathrm{P}\left\{\left|X_{n}\right|>C\right\}<\frac{\varepsilon}{2}+\frac{\varepsilon}{2}=\varepsilon .
\end{aligned}
$$

Таким образом, в нашем случае (2) эквивалентно $\left(2^{\prime}\right)$ :

$$
\forall \varepsilon>0 \quad \exists C_{\varepsilon} \quad \forall C \geqslant C_{\varepsilon} \quad \exists N_{C} \quad \forall n \geqslant N_{C}\left|A \int X_{n} d P-\int\left[X_{n}\right]^{C} d P\right|<\varepsilon .
$$

Теперь теорема 2 будет следовать из критерия перестановки 2-х пределов по базе, доказанного, например, в [3] (под базой здесь понимается непустая совокупность непустых множеств $\mathscr{W}$ такая, что $\left.\forall W_{1}, W_{2} \in \mathscr{W} \exists W \in \mathscr{W}, W \subseteq W_{1} \cap W_{2}\right)$.

Tеорема А. Пусть даны два множества $U$ и $V, \mathscr{U}-$ база в $U, \mathscr{V}-$ базав $V$, и функиия $h: U \times V \rightarrow \mathbb{R}$. И пусть существуют предель по каждой из баз: $f(u)=\lim _{\mathscr{V}} h(u, v) \quad \forall u \in \mathscr{U} u g(v)=\lim _{\mathcal{u}} h(u, v) \quad \forall v \in \mathscr{V}$. Тогда следующие условия әквивалентны:

1. предель $\lim _{\mathscr{U}} f(u)$ u $\lim _{\mathscr{V}} g(v)$ существуют и равны;

2 .

$$
\forall \varepsilon>0 \quad \exists S_{\varepsilon} \in \mathscr{U} \quad \forall u \in S_{\varepsilon} \quad \exists T_{u} \in \mathscr{V} \quad \forall v \in T_{u} \quad|h(u, v)-g(v)|<\varepsilon .
$$

Для доказательства теоремы 2 достаточно взять $U=V=\Omega, \mathscr{U}:=\{\{C \in \mathbb{R}$ : $\left.\left.C \geqslant C_{0}\right\}\right\}_{C_{0}=1}^{\infty}-$ база предела $C \rightarrow \infty, C \in \mathbb{R}, \mathscr{V}:=\{\{n \in \mathbb{N}: n \geqslant N\}\}_{N=1}^{\infty}-$ база предела $n \rightarrow \infty, N \in \mathbb{N}$, и $h(C, n):=\int\left[X_{n}\right]^{C} d P$. Используя лемму, видим, что

$$
f(C)=\lim _{n \rightarrow \infty} \int\left[X_{n}\right]^{C} d P=\int\left[X_{\infty}\right]^{C} d P
$$


по теореме Лебега,

$$
g(n)=\lim _{C \rightarrow \infty} \int\left[X_{n}\right]^{C} d P=(A) \int X_{n} d P
$$

в силу $A$-интегрируемости $X_{n}$. Тогда критерий перестановки пределов по базе даст нам, что (3), эквивалентное в нашем случае $\left(2^{\prime}\right)$, равносильно существованию $A$-интеграла предельной функции

$$
\lim _{C \rightarrow \infty} f(C)=\lim _{C \rightarrow \infty} \int\left[X_{\infty}\right]^{C} d P=(A) \int X_{\infty} d P
$$

и его равенство пределу интегралов $\lim _{n \rightarrow \infty} g(n)=\lim _{n \rightarrow \infty}(A) \int X_{n} d P$. Теорема доказана.

ДокАЗАТЕЛЬСтво теОремы 1 . Возьмем произвольное $B \in \bigcup_{n} \mathscr{F}_{n}$. Тогда последовательность случайных величин $\left\{\chi_{B} X_{n}\right\}_{n=1}^{\infty}$ удовлетворяет условиям теоремы 2 . В самом деле, так как $\mathrm{P}\left\{B \cap\left\{\left|X_{\infty}\right|>C\right\}\right\} \leqslant \mathrm{P}\left\{\left|X_{\infty}\right|>C\right\}$, то условие (1) теоремы 2 выполнено. Существует такое $M$, что $B \in \mathscr{F}_{M}$. Тогда для любого $m \geqslant M$ по определению мартингальной последовательности вьполнено $\int_{B} X_{m} d P=\int_{B} X_{M} d P$, а тогда первое утверждение теоремы 2 в этом случае равносильно тому, что

$$
\lim _{m \rightarrow \infty} \int_{B} X_{m} d P=\int_{B} X_{M} d P=\int_{B} X_{\infty} d P .
$$

Так как $B$ произвольно, это означает, что $X_{\infty}$ замькает мартингальную последовательность $\left\{X_{n}, \mathscr{F}_{n}\right\}$ справа. Второе из эквивалентньх утверждений теоремы 2 в нашем случае дает второе утверждение теоремы 1. Таким образом, теорема 1 доказана.

Теорема 1 дает существенно более общие достаточные условия замкнутости мартингальной последовательности справа, чем нижеследующая теорема В (это теорема 2 из [4]), сформулированная в терминах равномерной $A$-интегрируемости.

ОПрЕДЕЛЕниЕ 4 . Последовательность $A$-интегрируемых на множестве $B$ случайных величин $\left\{X_{n}\right\}$ называется равномерно $A$-интегрируемой на множестве $B$, если

$$
\sup _{n}\left|(A) \int_{B \cap\left\{\left|X_{n}\right|>C\right\}} X_{n} d P\right| \rightarrow 0 \quad \text { при } C \rightarrow \infty .
$$

ТЕОремА В. Пусть $\left\{X_{n}, \mathscr{F}_{n} ; n=1,2, \ldots\right\}$-мартингальная последовательность, сходящаяся почти всюду к случайной величине $X_{\infty}$. Пусть выполнены следующие условия:

1. $\mathrm{P}\left\{\left|X_{n}\right|>C\right\}=\overline{\bar{o}}(1 / C)$ при $C \rightarrow \infty$ равномерно по $n$;

2. последовательность $\left\{X_{n}\right\}$ равномерно $A$-интегрируема на каждом $B \in \bigcup_{n} \mathscr{F}_{n}$. Тогда $X_{\infty}$ является $A$-интегрируемой на каждом таком $B$ и служит последним әлементом для мартингальной последовательности $\left\{X_{n}, \mathscr{F}_{n}\right\}$.

В [1] был построен пример функции $f(x)$, являющейся последним элементом для мартингальной последовательности частных сумм ее ряда Фурье в смысле $A$-интеграла по 
системе Хаapa $\left\{X_{n}:=S_{2^{n}}(f, x), \mathscr{F}_{n}\right\}_{n=1}^{\infty}\left(\right.$ здесь $\mathscr{F}_{n}=\sigma\left(\Delta_{i}^{n}, i=0, \ldots, 2^{n}-1\right)$ - двоичные $\sigma$-алгебры, $\Delta_{i}^{n}=\left[i / 2^{n},(i+1) / 2^{n}\right), i=0, \ldots, 2^{n}-1,-$ двоичные интервалы ранга $n)$ :

$$
\begin{gathered}
f(x)=\left\{\begin{array}{rr}
0, & x \in\{0\} \cup \bigcup_{k=1}^{\infty}\left[\frac{1}{2^{2^{2 k}}}, \frac{1}{2^{2^{2 k}-1}}\right) \cup\left[\frac{1}{2}, 1\right], \\
\frac{2^{2^{2 k}+i}}{2^{2 k}} P_{n_{k, i}, m_{k}}\left(2^{2^{2 k}+i} x-1\right), & x \in\left[\frac{1}{2^{2^{2 k}+i}}, \frac{1}{2^{2^{2 k}+i-1}}\right), i=1, \ldots, 2^{2 k}, \\
n_{k, i}=2^{2 k}+i, m_{k}=2^{2 k+4}-2 k-1, \\
k=1,2, \ldots,
\end{array}\right. \\
P_{n, m}(x):=2 h_{0}^{1}(x)+\sum_{i=1}^{n-1} 2^{i} h_{0}^{i+1}(x)-\sum_{j=0}^{2^{m-1}-1} \sum_{i=1}^{n-1} 2^{i} h_{\left(2^{m-1}+j\right) 2^{i-1}}^{m+i}(x) ;
\end{gathered}
$$

здесь

$h_{0}(x):=h_{0}^{0}(x) \equiv 1, \quad h_{n}(x):=h_{j}^{i}(x)= \begin{cases}1, & x \in \Delta_{2 j}^{i}, \quad 0 \leqslant j \leqslant 2^{i-1}-1, \\ -1, & x \in \Delta_{2 j+1}^{i}, \quad i=1,2, \ldots, n=2^{i-1}+j,\end{cases}$ - ненормированные функции Хаара.

Но вместе с тем, как доказано в [1], любая подпоследовательность последовательности частных сумм $S_{n_{k}}(f, x)$ не является равномерно $A$-интегрируемой, и, таким образом, теорема В не может быть применена. Однако $\left\{X_{n}, \mathscr{F}_{n}\right\}$ удовлетворяет условиям теоремы 1. Покажем это.

Отрезок $\left[1 /\left(2^{2^{2 k+1}}\right), 1 /\left(2^{2^{2 k}}\right)\right)$ назовем $k$-м отрезком, $k=1,2, \ldots$ Назовем частную сумму $S_{n}(f, x)$ суммой типа $k$, если $N_{k+1}<n \leqslant N_{k+2}, N_{k}:=2^{2^{2 k}+1}+1$; только у таких частных сумм носитель имеет непустое пересечение с каждым из отрезков от 1 -го до $(k+1)$-го и сосредоточен на их объединении. Сужение частной суммы типа $k$ на каждый ее интервал постоянства, где она принимает ненулевое значение, назовем ступенькой типа $k$.

Пусть дано произвольное $\varepsilon, 0<\varepsilon<2$. Определим $C_{k}^{i}:=2^{2^{2 k+1}+2 i-2 k}$. Возьмем натуральное $K>\left(2-\log _{2} \varepsilon\right) / 2$. Положим $C_{\varepsilon}:=C_{K}^{1}$. Пусть $C \geqslant C_{\varepsilon}$. Тогда существуют натуральные числа

$$
i^{\prime}:=\left\{\begin{array}{ll}
i-1, & 2 \leqslant i \leqslant 2^{2 k} \\
2^{2(k-1)}, & i=1,
\end{array} \quad k^{\prime}:=\left\{\begin{array}{ll}
k, & 2 \leqslant i \leqslant 2^{2 k}, \\
k-1, & i=1,
\end{array} \quad k=1,2, \ldots,\right.\right.
$$

такие, что $C_{k^{\prime}}^{i^{\prime}}<C \leqslant C_{k}^{i}$. При этом $K \geqslant k$. Возьмем $N$ так, чтобы $S_{2^{N}}$ имела тип не менее $k+1$. Для произвольного $n \geqslant N$ наименьшее по модулю значение $X_{n}=S_{2^{n}}$, превосходящее $C$, будет $C_{k}^{i}$. Заметим, что $X_{n}$ совпадает на $k$-м отрезке с $f(x)$. Принимая это во внимание, при оценке $C \mathrm{P}\left\{\left|X_{n}\right|>C\right\}$ учтем на $k$-м отрезке все ступеньки типа $k$ частной суммы $S_{2^{n}}$, по модулю превосходящие $C$. Все же ступеньки на $1-\mathrm{M}, 2-\mathrm{м}, \ldots$, $(k-1)$-м отрезках по модулю не больше $C_{k^{\prime}}^{i^{\prime}}$. $\mathrm{Ha}(k+1)$-м, $(k+2)$-м, .. отрезках меру ступенек грубо оценим через $2^{-2^{2 k+2}}$, что больше чем сумма длин этих отрезков:

$$
\begin{gathered}
C \mathrm{P}\left\{\left|X_{n}\right|>C\right\} \leqslant C_{k}^{i} \mathrm{P}\left\{|f(x)| \geqslant C_{k}^{i}\right\} \leqslant 2 \cdot \frac{2^{2^{2 k+1}+2 i}}{2^{2 k}}\left(\sum_{j=i}^{2^{2 k}} \frac{1}{2^{2^{2 k+1}+2 j}}+\frac{1}{2^{2^{2 k+2}}}\right) \\
\leqslant 2^{2^{2 k+1}+2 i-2 k+1}\left(\frac{4 / 3}{2^{2^{2 k+1}+2 i}}\left(1-\frac{1}{2^{2^{2 k+1}-2 i+2}}\right)+\frac{1}{2^{2^{2 k+2}}}\right) \leqslant \frac{4}{2^{2 k}} \leqslant \frac{4}{2^{2 K}}<\varepsilon,
\end{gathered}
$$


т. е. 1-е условие теоремы 1 вьполнено.

Условие

$$
\begin{aligned}
& \left\{\forall B \in \bigcup_{n} \mathscr{F}_{n} \text { выполнено } \forall \varepsilon>0 \quad \exists C_{\varepsilon} \quad \forall C \geqslant C_{\varepsilon} \quad \exists N_{C} \quad \forall n \geqslant N_{C}\right. \\
& \left.\left|\int_{B \cap\left\{\left|X_{n}\right|>C\right\}} X_{n} d P\right|<\varepsilon\right\}
\end{aligned}
$$

устанавливается следующим образом (конечно, то, что оно здесь выполняется, напрямую следует из теоремы 2, учитьвая его необходимость, раз замкнутость справа была уже доказана в [1], однако для иллюстрации применения теоремы 2 в прямую сторону докажем его независимо, просто вычислением).

Ясно, что его достаточно проверить для отрезков, причем для $B$ вида $[a, b], a \neq 0$, оно очевидно. Если $B$ вида $[0, b]$, то $\forall \varepsilon>0 \exists C_{\varepsilon}=: C_{B}$ такая, что $\left\{x:|f(x)| \geqslant C_{\varepsilon}\right\} \subset[0, b]$. Взяв произвольное $C \geqslant C_{\varepsilon}$ для каждого $n \in \mathbb{N}$ определим множества $H_{n}:=\{x: 0<$ $\left.\left|X_{n}(x)\right| \leqslant C\right\}, H:=\{x: 0<|f(x)| \leqslant C\}$. Выберем $N_{C}$ настолько большим, что $H_{N_{C}}$ (а, следовательно, и все $H_{n}, n \geqslant N_{C}$ ) совпадают с $H$. При этом, как нетрудно видеть, $\forall n \geqslant N_{C} f(x)=X_{n}(x), x \in H$. Поэтому

$$
\begin{aligned}
\int_{B \cap\left\{\left|X_{n}\right|>C\right\}} X_{n} d P & =\int_{\left\{\left|X_{n}\right|>C\right\}} X_{n} d P=\int_{[0,1]} X_{n} d P-\int_{\left\{\left|X_{n}\right| \leqslant C\right\}} X_{n} d P \\
& =0-\int_{\{|f(x)| \leqslant C\}} f(x) d P=0 .
\end{aligned}
$$

Автор выражает благодарность профессору В. А. Скворцову за постановку задачи и ценные замечания.

\section{СПИСОК ЦИТИРОВАННОЙ ЛИТЕРАТУРЫ}

[1] Костин В. В., Скворцов В. А. Мартингальные последовательности в теории ортогональных рядов // Вестн. МГУ. (в печати).

[2] Колмогоров А.Н. Основные понятия теории вероятностей. М.: Физматгиз, 1936.

[3] Gordon R. A. An iterated limits theorem applied to the Henstok integral // Real Analysis Exchange. 1995/96. V. 21(2). P. 774-781.

[4] Skvortsov V.A. Martingale closure theorem for $A$-integrable martingal sequences // Real Analysis Exchange. (to appear). 